\title{
Phase 1 dose-escalation study of apatinib and irinotecan in esophageal squamous cell carcinoma patients
}

\author{
Jun Jia ${ }^{\#}$, Jing Yü, Zhiwei Sun, Ying Yang, Chuanling Liu, Yanjie Xiao, Xiaodong Zhang^^ \\ Key Laboratory of Carcinogenesis and Translational Research (Ministry of Education/Beijing), the VIP-II Gastrointestinal Cancer Division of the \\ Medical Department, Peking University Cancer Hospital \& Institute, Beijing, China \\ Contributions: (I) Conception and design: X Zhang; (II) Administrative support: X Zhang; (III) Provision of study materials or patients: All authors; \\ (IV) Collection and assembly of data: All authors; (V) Data analysis and interpretation: J Jia, J Yu; (VI) Manuscript writing: All authors; (VII) Final \\ approval of manuscript: All authors. \\ \#These authors contributed equally to this work. \\ Correspondence to: Dr. Xiaodong Zhang. No. 52 Fucheng Road, Haidian District, Beijing 100142, China. Email: zxd0829@hotmail.com.
}

Background: Apatinib, an inhibitor of vascular endothelial growth factor receptor (VEGFR), has been used to treat esophagogastric adenocarcinoma. However, the dosage of apatinib varies greatly in clinical practice, and its safety in esophageal squamous cell carcinoma (ESCC) patients is unclear. Therefore, we initiated a phase 1 dose-escalation trial to identify the maximum tolerated dose (MTD) of apatinib when combined with irinotecan in ESCC.

Methods: The trial had a standard 3+3 design. The dosage of irinotecan was fixed at $150 \mathrm{mg} / \mathrm{m}^{2}$ repeated every 2 weeks, while the daily dosage of apatinib was escalated from $250 \mathrm{mg}$, to $500 \mathrm{mg}$, to $750 \mathrm{mg}$. Doselimiting toxicity (DLT) was defined as grade 4 hematological or grade $3-4$ non-hematological adverse events (AEs).

Results: Twelve patients were enrolled. Three DLTs occurred, comprising a grade 3 perianal abscess and a grade 3 case of kaliopenia in the level 3 cohort, and a grade 4 leukopenia in the level 2 cohort. Based on these DLTs, the MTD of apatinib was $500 \mathrm{mg}$ daily. The most common AEs were leukopenia (91.7\%), fatigue (91.7\%), anemia (66.7\%), and diarrhea (58.3\%). One case of grade 2 hematochezia and one case of grade 2 subclavian vein thrombosis were observed. In the nine evaluable cases, the disease control rate (DCR) was $66.7 \%(6 / 9)$. The median progression-free and overall survival (OS) times were 3.6 \pm 1.2 and $6.6 \pm 3.4$ months, respectively.

Conclusions: This phase 1 dose-escalation trial showed that, when combined with irinotecan, a daily dose of $500 \mathrm{mg}$ apatinib was the optimum dose to treat ESCC.

Keywords: Apatinib; esophageal squamous cell carcinoma (ESCC); maximum tolerated dose (MTD)

Submitted Jul 09, 2020. Accepted for publication Dec 17, 2020.

doi: $10.21037 /$ tcr-20-2492

View this article at: http://dx.doi.org/10.21037/tcr-20-2492

\section{Introduction}

Esophageal cancer is the eighth most common cancer worldwide and the sixth most common cause of death from cancer (1). Unlike in western countries (where there is a higher incidence of adenocarcinoma), the majority of esophageal cancer in Asian countries is squamous cell carcinoma (SCC), which accounts for about $90 \%$ of esophageal cancers in Asia (2). Although there are several targeted cancer therapies, including ramucirumab [a monoclonal antibody against vascular endothelial growth

\footnotetext{
^ ORCID: Jun Jia, 0000-0002-1644-8075; Xiaodong Zhang, 0000-0001-9906-8555.
} 
factor receptor (VEGFR)] and trastuzumab (a monoclonal antibody against human epidermal growth factor receptor 2 ), that have been successfully used to treat esophageal and esophageal-gastric junction (EGJ) adenocarcinoma, neither monoclonal antibodies nor small kinase inhibitors have been shown to be effective for esophageal squamous cell carcinoma (ESCC).

Pathways associated vascular endothelial growth factor (VEGF) and its receptor VEGFR play an important role in the process of angiogenesis in tumors. Previous studies had been revealed that VEGF over-expressed in 24-93\% ESCC. Expression of VEGF was associated with microvessel density of ESCC tissue (3). VEGF was also reported of a prognostic factor of ESCC and a prospective predictive factor of chemoradiotherapy in ESCC (4). Thus, targeting VEGF or VEGFR is theoretically feasible to treat ESCC. Several double-blind, placebo-controlled, randomized phase 3 clinical trials have analyzed the efficacy and safety of anti-angiogenic agents in esophageal and/or gastric adenocarcinoma. Ramucirumab, as monotherapy (5) or combined with paclitaxel (6), significantly improved survival in patients with EGJ and gastric adenocarcinoma who had experienced standard treatment failure. In China, a selective anti-VEGFR2 tyrosine kinase inhibitor, apatinib, has been demonstrated to improve survival when used as a third-line treatment for EGJ and gastric adenocarcinoma $(7,8)$. The data from all these studies showed that antiVEGFR agents did not increase grade 3-4 hemorrhage in esophagogastric adenocarcinoma patients.

However, ESCC and esophageal adenocarcinoma are substantially different in their underlying etiological factors and tumorigenesis. Therefore, the success of antiangiogenic treatment in esophagogastric adenocarcinoma should not be extrapolated to ESCC without clinical trialbased evidence. A pilot study analyzed the efficacy and safety of apatinib in 62 ESCC patients who had experienced previous treatment failure (9). The outcomes were encouraging. The objective response rate (ORR) was $24.2 \%$ and the disease control rate (DCR) was $74.2 \%$. The median progression-free survival (PFS) and overall survival (OS) times were 115 and 209 days, respectively. The rate of grade 3-4 toxicities (including hand-foot syndrome, hypertension, proteinuria, hepatic injury, fatigue, esophagitis, and vomiting) was $59.7 \%$. There were no cases of fatal bleeding or esophageal fistula (9). However, the daily dosage of apatinib used in this previous study was $500 \mathrm{mg}$ instead of the $850 \mathrm{mg}$ that was indicated by the results of the phase 1 (10) and 2 (7) studies in gastric cancer. Furthermore, there were questions about the inadequate toxicity data and apatinib dosage used in the randomized phase III clinical trial of apatinib in esophagogastric adenocarcinoma $(11,12)$. Later studies in Chinese gastric cancer patients showed that the dosage of apatinib in real-world clinical practice varied wildly owing to toxicities $(13,14)$. The dosage of apatinib was reduced in later studies of apatinib in hepatic cancer (15), lung cancer (16), breast cancer (17), and ovarian cancer (18).

Thus, well-designed clinical trials are urgently needed to explore the optimum dosage and safety of apatinib in ESCC before determining its efficacy. Therefore, we designed a phase 1 clinical trial of apatinib combined with irinotecan as second-line treatment in patients with ESCC. Traditional chemotherapy is standard second-line treatment. Considering worsen performance status of patients who failed in 2nd-line treatment, only a few proportions of them had opportunity to receive further treatment. Thus, second-line treatment is the key to improve survival of ESCC patients. In order to improve the efficacy of secondline treatment and benefit patients, we used irinotecan in combination with apatinib instead of apatinib monotherapy as second-line treatment in this study. Although cisplatin, fluorouracil, and taxane are more frequently used to treat esophageal cancer, they are mostly used as first-line treatment. Irinotecan is a topoisomerase I inhibitor and has been demonstrated to be effective in esophageal cancer. Irinotecan monotherapy resulted in response rates of 10 $20 \%$ in advanced or metastatic esophageal cancer $(19,20)$. When combined with fluorouracil, docetaxel, or cisplatin, irinotecan also led to active and prolonged survival (21-23). Furthermore, irinotecan is recommended in the National Comprehensive Cancer Network (NCCN) guidelines as a preferred second-line treatment for esophageal cancer.

The pharmacokinetics of apatinib have been analyzed in a previous study of 46 cancer patients (10). Therefore, the present phase 1 clinical trial introduced a dose-escalation design, to identify the maximum tolerated dose (MTD) of apatinib. Phase I study play an extremely important role in the development of a cancer treatment. The principle of phase 1 study includes minimizing the numbers of patients allocated to ineffective or excessively cytotoxic agents, while identifying the best dose of a certain intervention or medicine. Although use of model-based Bayesian approaches had been increased recently in phase 1 study, traditional $3+3$ design is also widely used (24). And no single escalation method had been proven superior in all circumstances (25). The toxicities and maximum tolerable dosage of apatinib had been previous analyzed. The aim 
of prevent study was to identify the dosage of apatinib in a different disease, ESCC, when combined with irinotecan rather than to explore a totally new agent. Therefore, we introduced the traditional $3+3$ design in our study. We hope to identify a feasible daily dosage of apatinib for later clinical studies of ESCC. We present the following article in accordance with the MDAR checklist (available at http:// dx.doi.org/10.21037/tcr-20-2492).

\section{Methods}

\section{Patients}

The study was conducted in accordance with the Declaration of Helsinki (as revised in 2013). The study was approved by the ethics committee of Beijing Cancer Hospital (No. 2015YJZ25) and signed informed consent was taken from all the patients voluntarily before enrollment. The trial was also registered at ClinicalTrials. gov (NCT02645864) on January 5th, 2016.

Patients enrolled in this study had histologically confirmed recurrent ESCC with first-line treatment failure. First-line treatment was defined as first cytotoxic agents involved treatment, which included: (I) preoperative chemotherapy or chemoradiotherapy if disease progressed in 24 weeks after esophagectomy; (II) chemoradiotherapy if disease progressed in 24 weeks after treatment; (III) adjuvant chemotherapy if disease progressed in 24 weeks after treatment; (IV) first salvage chemotherapy for unresectable or metastatic disease.

The other main inclusion criteria were: age 18-70 years, an Eastern Cooperative Oncology Group (ECOG) performance status of $0-2$, a life expectancy $\geq 3$ months, measurable disease according to the Response Evaluation Criteria In Solid Tumors (RECIST) 1.1 criteria, normal electrocardiogram results, no history of congestive heart failure. Patients also had normal bone marrow, liver, and renal function, which were defined as: a hemoglobin level $\geq 100 \mathrm{~g} / \mathrm{L}$ (without blood transfusion within the previous 14 days); a platelet count $\geq 100 \times 10^{9} / \mathrm{L}$; a neutrophil count $\geq 2.0 \times 10^{9} / \mathrm{L}$; a creatinine level $\leq 1.5$ upper normal limitation (UNL) or a creatinine clearance rate $\geq 50 \mathrm{~mL} / \mathrm{min}$ (Cockcroft-Gault); a total bilirubin level $\leq 1.5 \mathrm{UNL}$; and aminotransferase $\leq 2.5 \mathrm{UNL}$ or $\leq 5 \mathrm{UNL}$ in cases liver metastasis. Patients should also have no bleeding or thrombosis disease. Normal coagulation function was required, including: activated partial thromboplastin time, prothrombin time, and international normalized ratio
(INR) each $\leq 1.5$ ULN. Female patients with childbearing potential agreed to contraception 1 week before treatment until 8 weeks after the last dose of the study drug. Male patients agreed to contraception before the first treatment until 8 weeks after the last dose of the study drug.

The exclusion criteria were as follows: patients who received irinotecan or apatinib previously; patients with uncontrolled hypertension (systolic pressure $\geq 140 \mathrm{mmHg}$ and diastolic pressure $\geq 90 \mathrm{mmHg}$ in case of antihypertensive drugs treatment); patients with grade $\geq 2$ coronary heart disease, uncontrolled arrhythmia, or QT interval prolongation (male $>450 \mathrm{~ms}$, women $>470 \mathrm{~ms}$ ); patients who were unable to take oral tablets for example serious vomiting, diarrhea, or intestinal obstruction; patients with abnormal coagulation function (INR $>1.5$ ULN etc.), thrombosis or receiving anticoagulant treatment; patients who currently had serious diseases and could not tolerate chemotherapy for example myocardial infarction, liver failure, or renal failure; patients with brain metastasis; and patients who with have bleeding risk, including: (I) melena or hematemesis in the previous 3 months; (II) concurrent peptic ulcer and fecal occult blood (++); or (III) fecal occult blood $(+)$ or $(+/-)$ and endoscopy revealed hemorrhagic diseases.

\section{Study design}

This phase 1 dose-escalation study used the traditional $3+3$ design. The dosage of irinotecan (Hengrui Pharmaceutical Co. Ltd. Lianyungang, China) was fixed at $150 \mathrm{mg} / \mathrm{m}^{2}$ and repeated every 2 weeks. The daily dosage of apatinib (Hengrui Pharmaceutical Co. Ltd.) was set at 3 levels: $250 \mathrm{mg}$ (level 1), $500 \mathrm{mg}$ (level 2), and $750 \mathrm{mg}$ (level 3). Dose escalation occurred when there was no dose-limiting toxicity (DLT) in all three patients or no more than one in six patients in the preceding cohort over three treatment cycles. If the disease did not progress after six cycles of combination treatment, apatinib was recommended for maintenance treatment until disease progression or intolerable adverse events (AEs).

Dose reduction of apatinib or irinotecan was not allowed. If there were any grade $\geq 2 \mathrm{AEs}$, a dose delay of no more than 2 weeks was allowed until all AEs disappeared or recovered to grade 1 . The DLT was defined as any grade 4 hematological AEs (including leukopenia, neutropenia, and thrombocytopenia), or any grade 3-4 non-hematological AEs in the first three treatment cycles. The optimum dosage of apatinib was identified by MTD while the 
Table 1 Patient characteristics and clinical response to irinotecan and apatinib treatment

\begin{tabular}{|c|c|c|c|c|c|c|c|c|c|}
\hline $\begin{array}{l}\text { Patient } \\
\text { No. }\end{array}$ & $\begin{array}{c}\text { Dosage of } \\
\text { apatinib (mg) }\end{array}$ & Gander & Age & $\begin{array}{l}\text { Location of } \\
\text { primary tumor }\end{array}$ & Metastatic sites & Cycles & Response & PFS & os \\
\hline 1 & 250 & M & 58 & Middle & $\begin{array}{l}\text { Supraclavicular, mediastinal, } \\
\text { celiac artery, and paraaortic LNs }\end{array}$ & 6 & SD & 8.9 & 10.2 \\
\hline 2 & 250 & M & 61 & Middle & Liver, paraaortic LNs & 3 & PD & 2.0 & 3.9 \\
\hline 3 & 250 & M & 64 & Middle & Lung, mediastinal LNs & 4 & SD & 2.8 & 3.7 \\
\hline 5 & 500 & M & 62 & Lower & Mediastinal LNs, local recurrence (stoma) & 6 & SD & 5.7 & 11.0 \\
\hline 6 & 500 & M & 49 & Lower & $\begin{array}{l}\text { Supraclavicular and mediastinal LNs, } \\
\text { adrenal gland, chest wall, abdominal wall }\end{array}$ & 6 & SD & 3.8 & 6.6 \\
\hline 10 & 500 & $M$ & 60 & Lower & $\begin{array}{l}\text { Mediastinal, hilar LNs, pleural and } \\
\text { peritoneal metastasis }\end{array}$ & 1 & - & - & - \\
\hline 11 & 500 & M & 55 & Lower & Lung, liver, paraaortic LNs & 3 & PD & 1.7 & 2.5 \\
\hline 12 & 500 & M & 61 & Lower & Lung, liver & 6 & SD & 3.6 & 3.9 \\
\hline
\end{tabular}

M, male; F, female; LNs, lymph nodes; SD, stable disease; PD, progressive disease; PFS, progression-free survival; OS, overall survival.

MTD was defined as the highest dose at which no more than one of six patients experienced a DLT event. AEs in the following treatment cycles (including maintenance treatment) were continuously recorded but not regarded as DLT. In case of grade 2-3 hematological or grade 2 nonhematological toxicity, the subsequent treatment cycle was temporarily delayed until all AEs recovered to grade 1. All AEs were treated according to clinical routines. For example, leukopenia was treated by granulocyte-colony stimulating factor and vomiting was treated by ondansetron and/or dexamethasone.

Computed tomography (CT) scans were obtained before treatment, every three cycles during treatment, and every 3 months during maintenance treatment and followup. Palliative radiotherapy for non-target lesions was allowed, to control symptoms after apatinib and irinotecan combination treatment.

\section{Statistical analysis}

The primary endpoints were the DLT and MDT, and the secondary endpoints were the ORR, median PFS, and median OS. The descriptive statistics involved calculating the means \pm standard errors. PFS and OS curves were plotted using Kaplan-Meier methods. SPSS 17.0 software (SPSS, Inc., Chicago, IL, USA) was used for the statistical analyses.

\section{Results}

Between Jan 2016 and July 2018, 12 patients were enrolled into the study. A 54-year-old man with metastatic ESCC in the level 2 cohort (apatinib $500 \mathrm{mg}$ ) withdrew from the study after one treatment cycle due to grade 2 fatigue. The safety data for this case were included. The median age was 57.1 years (range, 45-65 years). All patients had recurrent ESCC. All patients received previous chemotherapy involving taxane and platinum. Patient characteristics are summarized in Table 1.

\section{MTD and toxicities}

There was no dose reduction in any patients in the first three treatment cycles. The mean number of treatment 
Table 2 Adverse events of all 12 patients enrolled according to Common Terminology Criteria Adverse Events (CTC AE) version 4.0

\begin{tabular}{|c|c|c|c|c|c|c|c|c|c|c|c|c|c|c|c|c|c|c|c|c|c|c|}
\hline $\begin{array}{l}\text { Patient } \\
\text { No. }\end{array}$ & $\begin{array}{c}\text { Dosage of } \\
\text { apatinib (mg) }\end{array}$ & Leukopenia & Neutropenia & Thrombocytopenia & Anemia & Hemorrhage & $\begin{array}{l}\text { Venous } \\
\text { thrombosis }\end{array}$ & Infection & $\begin{array}{c}\text { Alanine } \\
\text { aminotransferase }\end{array}$ & Bilirubin & Kaliopenia & $\begin{array}{l}\text { Nausea/ } \\
\text { vomiting }\end{array}$ & Apositia & Diarrhea & $\begin{array}{c}\text { Muscular } \\
\text { Pain }\end{array}$ & Proteinuria & Hypertension & Rash & Fatigue & $\begin{array}{l}\text { Loss of } \\
\text { weight }\end{array}$ & $\begin{array}{l}\text { Peripheral } \\
\text { neuritis }\end{array}$ & Alopecia \\
\hline 1 & 250 & 2 & 2 & 0 & 1 & 0 & 0 & 0 & 2 & 0 & 0 & 0 & 0 & 0 & $1^{a}$ & 1 & 0 & 0 & 1 & 0 & 0 & 0 \\
\hline 2 & 250 & 3 & 3 & 1 & 0 & 0 & 0 & 0 & 1 & 1 & 0 & 1 & 1 & 1 & 1 & 0 & 0 & 0 & 1 & 0 & 1 & 0 \\
\hline 3 & 250 & 2 & 2 & 0 & 2 & 0 & 2 & 0 & 0 & 0 & 0 & 0 & 1 & 1 & 1 & 0 & 0 & 0 & 2 & 1 & 1 & 0 \\
\hline 4 & 500 & 1 & 0 & 0 & 0 & 0 & 0 & 0 & 1 & 0 & 0 & 0 & 2 & 0 & 0 & 2 & 0 & 0 & 1 & 1 & 0 & 0 \\
\hline 5 & 500 & 3 & 3 & 1 & 2 & 0 & 0 & 0 & 0 & 0 & 0 & 0 & 1 & 0 & 0 & 0 & 1 & 0 & 1 & 0 & 0 & 0 \\
\hline 6 & 500 & 2 & 3 & 0 & 1 & 0 & 0 & 0 & 0 & 1 & 0 & 2 & 1 & 1 & 0 & 1 & 0 & 0 & 2 & 0 & 0 & 0 \\
\hline 7 & 750 & 3 & 3 & 2 & 1 & 0 & 0 & 0 & 0 & 2 & 3 & 0 & 0 & 2 & 0 & 0 & 0 & 0 & 0 & 0 & 0 & 0 \\
\hline 8 & 750 & 0 & 0 & 0 & 0 & 0 & 0 & $3^{b}$ & 0 & 0 & 0 & 1 & 0 & 1 & 0 & 0 & 0 & 0 & 1 & 0 & 0 & 0 \\
\hline 9 & 500 & $4^{\circ}$ & 3 & 2 & 2 & 0 & 0 & 0 & 0 & 0 & 0 & 2 & 1 & 1 & 1 & 1 & 0 & 1 & 1 & 0 & 0 & 1 \\
\hline 10 & 500 & 3 & 2 & 0 & 1 & 0 & 0 & 0 & 0 & 0 & 1 & 0 & 0 & 3 & 0 & 2 & 0 & 0 & 2 & 0 & 0 & 0 \\
\hline 11 & 500 & 2 & 1 & 0 & 3 & 2 & 0 & 0 & 1 & 1 & 0 & 0 & 1 & 0 & 0 & 0 & 0 & 0 & 2 & 0 & 0 & 0 \\
\hline 12 & 500 & 2 & 1 & 1 & 0 & 0 & 0 & 0 & 0 & 0 & 1 & 1 & 0 & 0 & 1 & 0 & 0 & 0 & 2 & 0 & 0 & 0 \\
\hline
\end{tabular}


cycles was 4.3 (range, 3-6) in the level 1 cohort (apatinib $250 \mathrm{mg}$ ) and level 2 cohort (apatinib $500 \mathrm{mg}$ ). However, there was a mean dose delay of 2.0 days per treatment cycle in the level 1 cohort (apatinib $250 \mathrm{mg}$ ) and level 2 cohort (apatinib $500 \mathrm{mg}$ ). In the level 3 cohort (apatinib $750 \mathrm{mg}$ ), two patients were enrolled and each of them received only one treatment cycle. Treatment was then stopped due to grade 3 non-hematological toxicities and there was no dose delay.

In the dose-escalation period, three patients were enrolled in the level 1 cohort (apatinib $250 \mathrm{mg}$ ). As none of them developed DLT, the next three patients were enrolled in the level 2 cohort (apatinib $500 \mathrm{mg}$ ) and no DLT was reported. According to the study design, the level 3 cohort (apatinib $750 \mathrm{mg}$ ) then started. Two patients were enrolled in this cohort and both of them reported DLTs. Patient \#7, a 65 -year-old woman, presented with grade 3 kaliopenia with grade 2 diarrhea and grade 3 leukopenia during the first treatment cycle. Patient \#8, a 52-year-old man, presented with a grade 3 infection (perianal abscess) and grade 1 diarrhea but without leukopenia during the first treatment cycle. This led to the closure of the level 3 cohort and the expansion of the level 2 cohort to six patients. One case of DLT was then observed in the level 2 cohort. Patient \#9, a 45-year-old man, presented with grade 4 leukopenia and grade 2 fever. No further DLTs occurred in the following patients in the level 2 cohort. The MTD of apatinib was determined to be $500 \mathrm{mg}$ daily when accompanied with $150 \mathrm{mg} / \mathrm{m}^{2}$ irinotecan repeated every 2 weeks.

As shown in Table 2, the most common AEs were leukopenia $(91.7 \%)$, fatigue $(91.7 \%)$, anemia $(66.7 \%)$, diarrhea $(58.3 \%)$, apositia $(58.3 \%)$, vomiting $(41.7 \%)$, and thrombocytopenia (41.7\%). Additionally, one case of grade 2 hematochezia and one case of grade 2 subclavian vein thrombosis were observed. Patient \#11, a 55-yearold man with ESCC and lung metastasis, presented with grade 2 hematochezia during the third treatment cycle. There was fresh blood in his stool intermittently, with a volume of about $20-30 \mathrm{~mL}$. The patient had no history of hemorrhoids, and digital rectal examination was negative. Colonoscopy was suggested but the patient refused and declined further treatment due to disease progression. No further hemorrhoids occurred during follow-up. Patient \#3, a 64-year-old man, reported left upper limb pain during the fourth treatment cycle. Ultrasonic examination confirmed grade 2 subclavian vein thrombosis. The apatinib treatment was discontinued and low-molecular-weight heparin calcium treatment was started. The subclavian vein thrombosis was stable during follow-up. One case of hypertension and one case of rash were also observed among the subjects.

\section{Clinical response}

Among the 12 patients, patients \#8 and \#9 discontinued treatment due to DLTs. Patient \#10 withdrew from the study after one treatment cycle. Although patient \#7, a 65-year-old woman, reported DLTs (grade 3 kaliopenia and grade 2 diarrhea) in the first treatment cycle. UGT1A1*28 assessment showed 6/7 polymorphism. After communicating with the patient, she continued apatinib treatment with a reduction of irinotecan and finished three treatment cycles. Among the nine evaluable patients, four patients completed six treatment cycles and two of them received daily maintenance treatment of $250 \mathrm{mg}$ apatinib.

As shown in Table 1 and Figure 1, there were no cases involving complete response (CR) or partial response (PR). Six cases of stable disease (SD) were observed, and the DCR was $66.7 \%$. Patients \#1 and \#3 in the apatinib 250 $\mathrm{mg}$ cohort, patients \#5, \#6, and \#12 in the apatinib 500 $\mathrm{mg}$ cohort, and patient \#7 in the apatinib $750 \mathrm{mg}$ cohort had SD after three treatment cycles (Figure 2). Patient \#2 in the apatinib $250 \mathrm{mg}$ cohort and patients \#4 and \#11 in the apatinib $500 \mathrm{mg}$ cohort had progressive disease (PD) after three treatment cycles. In patient \#12, cavitation of lung metastatic tumors was observed, though the treatment response was SD according to the RECIST 1.1 criteria. After a median follow-up of 8.4 months, there were eight cases of PD and seven deaths among the nine evaluable

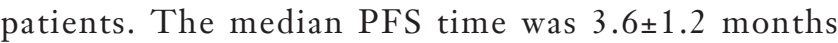
(range, 1.6-8.9 months) and the median OS time was $6.6 \pm 3.4$ months (range, $2.2-10.2$ months).

\section{Discussion}

VEGF and its receptor VEGFR are the principal mediators of the angiogenic pathway. Among the VEGFRs, VEGFR2 is a key factor. Activation of VEGFR2 promotes proliferation and migration of vascular endothelial cells and also increases cellular permeability, which are necessary for angiogenesis (3). Therefore, more attention should be paid to bleeding and thromboembolic events.

In a previous study of ramucirumab in non-small cell lung cancer, both patients with adenocarcinoma and patients with SCC were recruited. The results showed that compared with placebo, the rate of any-grade hemorrhage was higher in the ramucirumab arm (29\% versus $15 \%)$, 


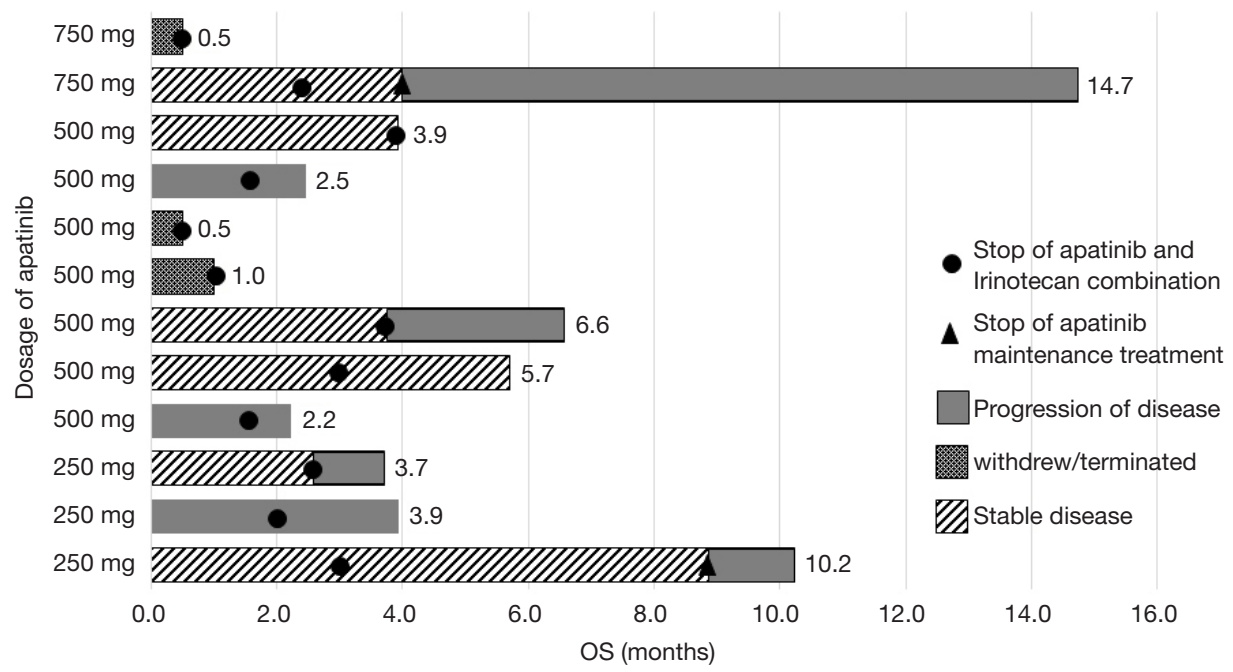

Figure 1 Response of all 12 patients to apatinib and irinotecan combination treatment. Totally 12 patients enrolled. Patients \#1 and \#3 in the apatinib $250 \mathrm{mg}$ cohort, patients \#5, \#6, and \#12 in the apatinib $500 \mathrm{mg}$ cohort, and patient \#7 in the apatinib $750 \mathrm{mg}$ cohort had stable disease (SD) after three treatment cycles. The disease control rate (DCR) was 66.7\% (6/9) in 9 evaluable patients. After a median followup of 8.4 months, there were eight cases of progressive disease (PD) and seven deaths. The median progression-free survival (PFS) time was $3.6 \pm 1.2$ months and the median overall survival (OS) time was $6.6 \pm 3.4$ months.

while the rate of serious bleeding of grade $\geq 3$ was similar ( $2 \%$ versus $2 \%$ ) (26). In a meta-analysis of safety data from six randomized, placebo-controlled trials of ramucirumab, the rate of any-grade bleeding in the ramucirumab arm was also higher than that in the placebo arm $[37.5 \%$ versus $19.0 \%$, relative risk (RR): 2.0, 95\% CI, 1.8-2.2]. Grade 1-2 epistaxis was the most frequently reported bleeding event in the ramucirumab arm. Grade $\geq 3$ bleeding was similar in the ramucirumab arm $(2.7 \%)$ and control arm $(2.8 \%$; RR: $1.1,95 \%$ CI, 0.8-1.5) (27).

It was reported that apatinib did not increase the risk of bleeding in a randomized phase 3 clinical trial of esophagogastric adenocarcinoma patients. The rate of any-grade bleeding was $19.9 \%$ and the rate of grade $3-4$ bleeding was $3.4 \%$ in the apatinib arm, compared with $24.2 \%$ and $7.7 \%$ in the placebo arm (8). No serious bleeding events were observed. In a study of 62 ESCC patients who received apatinib treatment, it was reported that there were no bleeding events (9). However, there have been case reports showing that apatinib resulted in fatal gastrointestinal hemorrhage in gastric cancer (28) and hemoptysis in advanced esophageal cancer treated with apatinib (29). In the present study, one case of grade 2 hematochezia was observed. The patient had fresh blood in his stool intermittently, with a volume of about 20-30 $\mathrm{mL}$, in the third treatment cycle. We suspected that the bleeding occurred in the colon, as digital rectal examination indicated no hemorrhoids and the patient had no upper gastrointestinal tract symptoms. Unfortunately, the patient refused to accept a colonoscopy examination. In the present study, all enrolled patients had recurrent ESCC after esophagectomy. Thus, the hemorrhage risk of apatinib in ESCC patients whose primary tumor has not been resected remains unclear. Further investigation is needed to explore the bleeding risk of apatinib in ESCC patients.

The other side effect of anti-angiogenic agents is the risk of thromboembolic events. In a pooled analysis of patients in randomized phase II and III studies of bevacizumab (a monoclonal antibody against VEGF-A), the incidence of any-grade venous thromboembolic events (VTE) was not significantly increased in the bevacizumab arms compared with the control arms (10.9\% versus $9.8 \%$, odds ratio 1.14 , $95 \% \mathrm{CI}, 0.96-1.35, \mathrm{P}=0.13)$. The incidence of grade $3-5$ events was also similar in both groups (30). In a metaanalysis of ramucirumab (involving 11 clinical trials), the risk rate of any-grade thromboembolic events was 0.97 (95\% CI, 0.62-1.52, $\mathrm{P}=0.91)$ in the ramucirumab arms and 0.83 (95\% CI, 0.52-1.35, P=0.46) in the control arms, with no significant difference (31).

The incidence of thromboembolic events was not reported in the phase 2 and 3 clinical trials of apatinib in gastric cancer $(7,8)$. In another study of apatinib in 62 

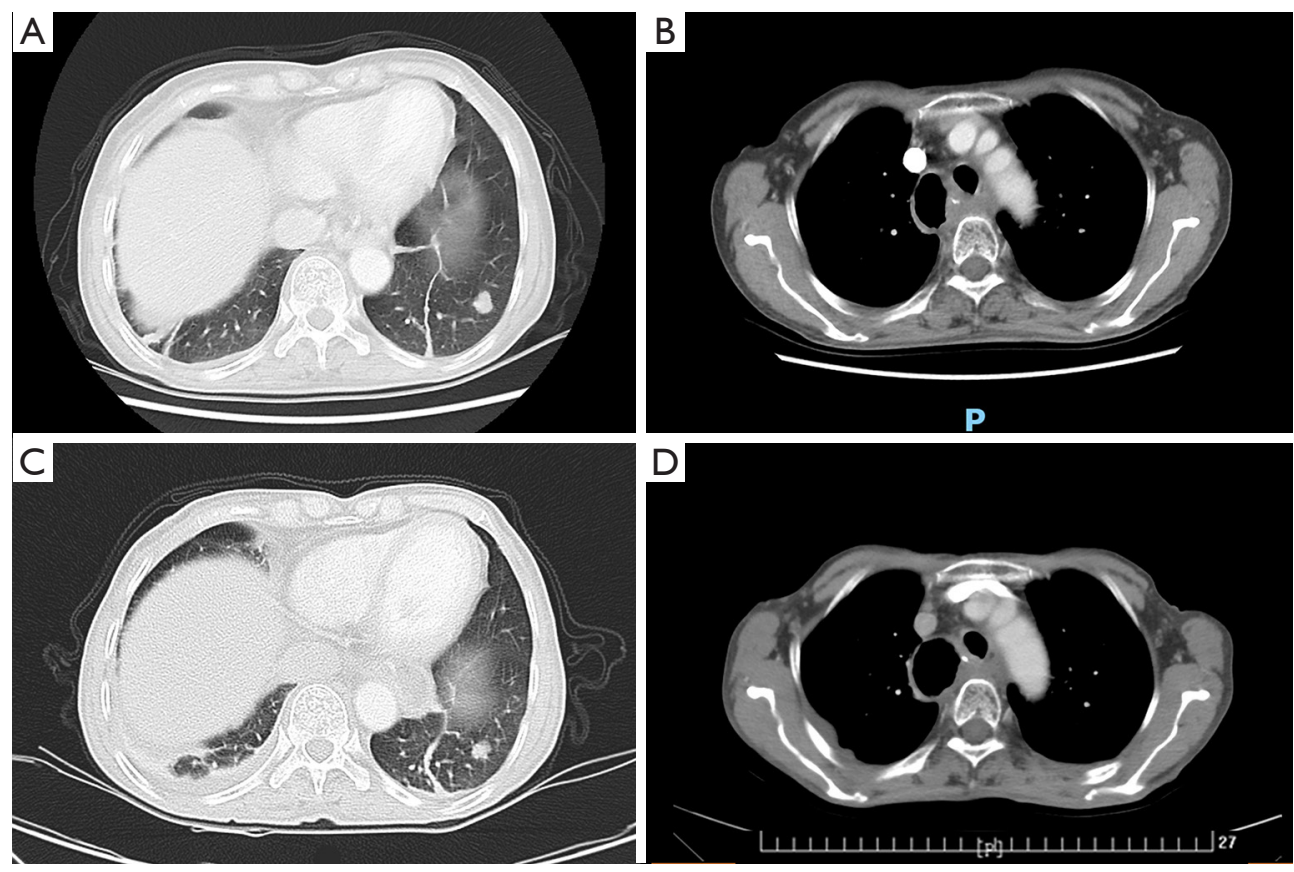

Figure 2 Response of combination treatment of apatinib with irinotecan in the \#7 patient. The \#7 patient had lung (A) and mediastinal lymph node (B) metastasis. She was given combination treatment of apatinib $750 \mathrm{mg}$ daily and irinotecan $150 \mathrm{mg} / \mathrm{m}^{2}$ every $2 \mathrm{weeks}$ for three cycles. Both lung (C) and mediastinal lymph node (D) lesions shrunk after treatment but fail to meet partial response of Response Evaluation Criteria In Solid Tumors (RECIST) 1.1 criteria and was identify as stable disease.

ESCC patients, the incidence of thromboembolic events was also not reported (9). However, in a later study of 29 ovarian cancer patients who received apatinib, four cases of VTE including one case of grade 3 VTE were observed (18). In the present study, one case of grade 2 subclavian vein thrombosis was observed, which indicates that apatinib might also result in thromboembolic events.

In the present study, the most common AEs were leukopenia, fatigue, anemia, diarrhea, apositia, vomiting, and thrombocytopenia. These AEs were also reported in previous studies of apatinib $(7,8)$ and irinotecan $(21,22)$. The high incidence of hematologic toxicities in the present study might be due to the combination of apatinib with the cytotoxic agent irinotecan. Unlike in previous reports (7-9), there were no cases of hand-foot syndrome, only one case of proteinuria, and only one case of hypertension in the present study, which were much lower rates than the rates in previous reports. In a phase 3 trial of gastric cancer, the incidence of hypertension, proteinuria, and hand-foot syndrome was $35.2 \%, 47.7 \%$, and $27.8 \%$, respectively, in the apatinib group (8).

Previous studies showed that irinotecan monotherapy resulted in an ORR of $10-15 \%$ in $\operatorname{ESCC}(19,20)$. As a monotherapy for ESCC, it was reported that apatinib resulted in an ORR of $24.2 \%$ and a DCR of $74.2 \%$. The median PFS and OS were 115 and 209 days respectively (9). In the present study, CR and PR were not observed, and the DCR was $66.7 \%(6 / 9)$. However, we observed one case of lung metastatic tumor cavitation in one ESCC patient after apatinib treatment. Previous research revealed that cavitation of tumor lesions is a characteristic of antiangiogenic agents. In the present study, after a median follow-up of 8.4 months, the median PFS time was $3.6 \pm 1.2$ months and the median OS time was $6.6 \pm 3.4$ months, which was similar to the previous results. Based on data of previous studies and present study, we believed that, as an anti-angiogenetic agent, apatinib had potential anti-tumor activity and might be used to treat ESCC in the future.

In conclusion, the present study identified the MDT of apatinib as $500 \mathrm{mg}$ daily when combined with irinotecan. However, when combined with other cytotoxic agents, the dosage of apatinib might be changed. Cases of bleeding and VTE were observed and further investigations are urgently needed to explore these risks of apatinib. A phase 2 study 
with a larger sample size has been carried out to explore the safety and efficacy of apatinib in ESCC.

\section{Acknowledgments}

An abstract on the design of this study was presented as a poster (under the title "Apatinib and irinotecan combination treatment in recurrent first-line chemotherapy refractory esophageal squamous cell carcinoma: a phase I doseescalation study") at the ESMO 2017 Annual Congress. The poster was published in "Poster Abstracts" in the Annals of Oncology under the title "781TiP Apatinib and irinotecan combination treatment in first-line chemotherapy refractory esophageal squamous cell carcinoma: A phase I dose-escalation study" (https://doi.org/10.1093/annonc/ $\operatorname{mdx} 369.163)$.

Funding: This study was funded by the Chinese Society of Clinical Oncology (CSCO)-Hengrui Cancer Research Foundation (2016).

\section{Footnote}

Reporting Checklist: The authors have completed the MDAR checklist. Available at http://dx.doi.org/10.21037/tcr-202492

Data Sharing Statement: Available at http://dx.doi. org/10.21037/tcr-20-2492

Peer Review File: Available at http://dx.doi.org/10.21037/ tcr-20-2492

Conflicts of Interest: All authors have completed the ICMJE uniform disclosure form (available at http://dx.doi. org/10.21037/tcr-20-2492). The authors have no conflicts of interest to declare.

Ethical Statement: The authors are accountable for all aspects of the work in ensuring that questions related to the accuracy or integrity of any part of the work are appropriately investigated and resolved. The study was conducted in accordance with the Declaration of Helsinki (as revised in 2013). The study was approved by the ethics committee of Beijing Cancer Hospital (No. 2015YJZ25). Signed informed consent was voluntarily provided by all patients before enrollment. The trial was also registered at ClinicalTrials.gov (registration no. NCT02645864).
Open Access Statement: This is an Open Access article distributed in accordance with the Creative Commons Attribution-NonCommercial-NoDerivs 4.0 International License (CC BY-NC-ND 4.0), which permits the noncommercial replication and distribution of the article with the strict proviso that no changes or edits are made and the original work is properly cited (including links to both the formal publication through the relevant DOI and the license). See: https://creativecommons.org/licenses/by-ncnd/4.0/.

\section{References}

1. Torre LA, Bray F, Siegel RL, et al. Global cancer statistics, 2012. CA Cancer J Clin 2015;65:87-108.

2. Arnold M, Soerjomataram I, Ferlay J, et al. Global incidence of oesophageal cancer by histological subtype in 2012. Gut 2015;64:381-7.

3. Kleespies A, Guba M, Jauch KW, et al. Vascular endothelial growth factor in esophageal cancer. J Surg Oncol 2004;87:95-104.

4. Peng J, Shao N, Peng H, et al. Prognostic significance of vascular endothelial growth factor expression in esophageal carcinoma: a meta-analysis. J BUON 2013;18:398-406.

5. Fuchs CS, Tomasek J, Yong CJ, et al. Ramucirumab monotherapy for previously treated advanced gastric or gastro-oesophageal junction adenocarcinoma (REGARD): an international, randomised, multicentre, placebocontrolled, phase 3 trial. Lancet 2014;383:31-9.

6. Wilke H, Muro K, Van Cutsem E, et al. Ramucirumab plus paclitaxel versus placebo plus paclitaxel in patients with previously treated advanced gastric or gastrooesophageal junction adenocarcinoma (RAINBOW): a double-blind, randomised phase 3 trial. Lancet Oncol 2014;15:1224-35.

7. Li J, Qin S, Xu J, et al. Apatinib for chemotherapyrefractory advanced metastatic gastric cancer: results from a randomized, placebo-controlled, parallel-arm, phase II trial. J Clin Oncol 2013;31:3219-25.

8. Li J, Qin S, Xu J, et al. Randomized, Double-Blind, Placebo-Controlled Phase III Trial of Apatinib in Patients With Chemotherapy-Refractory Advanced or Metastatic Adenocarcinoma of the Stomach or Gastroesophageal Junction. J Clin Oncol 2016;34:1448-54.

9. Li J, Wang L. Efficacy and safety of apatinib treatment for advanced esophageal squamous cell carcinoma. Onco Targets Ther 2017;10:3965-9. 
10. Li J, Zhao X, Chen L, et al. Safety and pharmacokinetics of novel selective vascular endothelial growth factor receptor-2 inhibitor YN968D1 in patients with advanced malignancies. BMC Cancer 2010;10:529.

11. Zhang S. Problematic Analysis and Inadequate Toxicity Data in Phase III Apatinib Trial in Gastric Cancer. J Clin Oncol 2016;34:3821.

12. Fornaro L, Vasile E, Falcone A. Apatinib in Advanced Gastric Cancer: A Doubtful Step Forward. J Clin Oncol 2016;34:3822-3.

13. Zhang Y, Han C, Li J, et al. Efficacy and safety for Apatinib treatment in advanced gastric cancer: a real world study. Sci Rep 2017;7:13208.

14. Ruan H, Dong J, Zhou X, et al. Multicenter phase II study of apatinib treatment for metastatic gastric cancer after failure of second-line chemotherapy. Oncotarget 2017;8:104552-9.

15. Lu W, Jin XL, Yang C, et al. Comparison of efficacy between TACE combined with apatinib and TACE alone in the treatment of intermediate and advanced hepatocellular carcinoma: A single-center randomized controlled trial. Cancer Biol Ther 2017;18:433-8.

16. Xu J, Liu X, Yang S, et al. Clinical response to apatinib monotherapy in advanced non-small cell lung cancer. Asia Pac J Clin Oncol 2018;14:264-9.

17. Hu X, Zhang J, Xu B, et al. Multicenter phase II study of apatinib, a novel VEGFR inhibitor in heavily pretreated patients with metastatic triple-negative breast cancer. Int J Cancer 2014;135:1961-9.

18. Miao M, Deng G, Luo S, et al. A phase II study of apatinib in patients with recurrent epithelial ovarian cancer. Gynecol Oncol 2018;148:286-90.

19. Mühr-Wilkenshoff F, Hinkelbein W, Ohnesorge I, et al. A pilot study of irinotecan (CPT-11) as single-agent therapy in patients with locally advanced or metastatic esophageal carcinoma. Int J Colorectal Dis 2003;18:330-4.

20. Burkart C, Bokemeyer C, Klump B, et al. A phase II trial of weekly irinotecan in cisplatin-refractory esophageal cancer. Anticancer Res 2007;27:2845-8.

21. Williamson SK, McCoy SA, Gandara DR, et al. Phase II trial of gemcitabine plus irinotecan in patients with esophageal cancer: a Southwest Oncology Group (SWOG) trial. Am J Clin Oncol 2006;29:116-22.

22. Kim M, Keam B, Kim TM, et al. Phase II Study of Irinotecan and Cisplatin Combination Chemotherapy in Metastatic, Unresectable Esophageal Cancer. Cancer Res Treat 2017;49:416-22.

23. Zhao H, Owen S, Davies EL, et al. The Effect of Aurora Kinase Inhibitor on Adhesion and Migration in Human Breast Cancer Cells and Clinical Implications. World J Oncol 2017;8:151-61.

24. Jaki T, Clive S, Weir CJ. Principles of dose finding studies in cancer: a comparison of trial designs. Cancer Chemother Pharmacol 2013;71:1107-14.

25. Hansen AR, Graham DM, Pond GR, et al. Phase 1 trial design: is $3+3$ the best? Cancer Control 2014;21:200-8.

26. Garon EB, Ciuleanu TE, Arrieta O, et al. Ramucirumab plus docetaxel versus placebo plus docetaxel for secondline treatment of stage IV non-small-cell lung cancer after disease progression on platinum-based therapy (REVEL): a multicentre, double-blind, randomised phase 3 trial. Lancet 2014;384:665-73.

27. Arnold D, Fuchs CS, Tabernero J, et al. Meta-analysis of individual patient safety data from six randomized, placebo-controlled trials with the antiangiogenic VEGFR2-binding monoclonal antibody ramucirumab. Ann Oncol 2017;28:2932-42.

28. Li XF, Tan YN, Cao Y, et al. A Case Report of Gastrointestinal Hemorrhage and Perforation During Apatinib Treatment of Gastric Cancer. Medicine (Baltimore) 2015;94:e1661.

29. Wang W, Zhang L, Xie Y, et al. Fatal hemoptysis in patients with advanced esophageal cancer treated with apatinib. Onco Targets Ther 2018;11:2565-70.

30. Hurwitz HI, Saltz LB, Van Cutsem E, et al. Venous thromboembolic events with chemotherapy plus bevacizumab: a pooled analysis of patients in randomized phase II and III studies. J Clin Oncol 2011;29:1757-64.

31. Abdel-Rahman O, ElHalawani H. Risk of cardiovascular adverse events in patients with solid tumors treated with ramucirumab: A meta analysis and summary of other VEGF targeted agents. Crit Rev Oncol Hematol 2016;102:89-100.
Cite this article as: Jia J, Yu J, Sun Z, Yang Y, Liu C, Xiao Y, Zhang X. Phase 1 dose-escalation study of apatinib and irinotecan in esophageal squamous cell carcinoma patients. Transl Cancer Res 2021;10(2):627-636. doi: 10.21037/tcr-20-2492 